\title{
An Adaptive Observer Design for Nonlinear Systems Affected by Unknown Disturbance with Simultaneous Actuator and Sensor Faults. Application to a CSTR
}

\author{
Sana Bzioui ${ }^{1, *(\mathbb{D})}$, Rafik Channa ${ }^{1}$ \\ 1 LAEPT Laboratory, Department of Physics. Cadi Ayyad University. Marrakech, Morocco \\ * Correspondence: bzioui.sana@gmail.com (S.B.);
}

Scopus Author ID 57201718094

Received: 6.08.2021; Revised: 20.09.2021; Accepted: 24.09.2021; Published: 16.10.2021

\begin{abstract}
Continuous Stirred Tank Reactor (CSTR) is an important system in the chemical and biological industries. It's characterized by a complex nonlinear behavior and is usually affected by faults and disturbances. Therefore, the states and faults estimation of a CSTR is always a challenging task for automated process researchers and engineers. This paper proposes an adaptive observer. This paper proposes an adaptive observer in order to estimate states and actuator and sensor faults simultaneously under unknown disturbance. Firstly, the approach of the Takagi-Sugeno multi-model is proposed to transform the complex nonlinear model into several simple linear sub-models. However, the states of the considered isotherm CSTR are not completely measurable, so the multi-model is represented with non-measurable premise variables. Then, in order to transform the considered system into a system with an unknown input, a mathematical transformation is introduced to describe the sensor faults as actuator faults. The proposed observer is designed, and the exponential stability conditions are studied with the Lyapunov theory and L2 optimization and formulated in terms of linear matrix inequalities. Finally, to improve the effectiveness of the proposed observer, a numerical simulation is carried out on a CSTR.
\end{abstract}

Keywords: CSTR; nonlinear system; multi-model approach; state estimation; faults estimation; adaptive observer; actuator fault; sensor fault.

(C) 2021 by the authors. This article is an open-access article distributed under the terms and conditions of the Creative Commons Attribution (CC BY) license (https://creativecommons.org/licenses/by/4.0/).

\section{Introduction}

Continuous Stirred Tank Reactor is used in biochemical and pharmaceutical processes in water treatment and several applications due to its appropriate mixing property. It is used for the perfect mixing of chemical components that are continuously added to the reactor in the chemical industry. The CSTR is characterized by complex nonlinear behavior and is exposed to faults and disturbances.

The complete knowledge of a system's state is necessary to construct a control law or a diagnosis mechanism. But, the state cannot be directly measurable due to economic or technical reasons. Therefore, state estimation becomes a principal step in process engineering. Observers have been used to generate a state estimation. An observer is based on the input and the measurable output to obtain an estimate of the state. The observer has first been proposed by Luenberger [1] for linear systems and later constructed for nonlinear systems [2].

Various approaches have been proposed to ensure the state estimation of a CSTR. In [3], a state estimation of a chemical reactor is proposed using the Extended Kalman Filter (EKF), Particle Filter (PF), and recently developed variational bayesian filter (VBF). In [4], the 
Extended Kalman Filter (EKF) and the neural network (NN) based state filtering algorithm has been used to estimate the states variables of a CSTR. In [5], the states of a CSTR are estimated using Luenberger Observer, Sliding Mode Observers (SMO), and Kalman Observers (KO). However, these works are based directly on the nonlinear model of the CSTR, and from the mathematical point of view, an observed design based directly on the nonlinear model is very complex due to the high nonlinearity of the system behavior. The best solution to this problem is the Takagi-Sugeno (TS) multi-model approach [6]. It consists of representing the nonlinear model as an interpolation of simple linear models; each submodel presents the system's behavior on a limited part of the operating space.

In industry, the CSTR is usually exposed to faults, affecting the system's input as an actuator fault or the system's output as a sensor fault. In practice, actuator faults and sensor faults can occur simultaneously. Thus, the fault sensor may destroy the actuator due to the wrong measurement. For that purpose, the control and diagnosis framework requires knowledge of all the system states and the faults affecting them. Consequently, an observer is important to estimate these signals simultaneously.

Many works have been proposed observers for state estimation of a TS system under faults [7-24]. We can note some applications on chemical systems using the Fuzzy Luenberger observer [25,26], the Proportional-Integral observer (PIO) [27-29], the Unknown Input Observer (UIO) [30,31], the Hळ observer [32,33], and sliding mode observer [34]. Most of these works supposed that the variable premises are measurable or coped with actuator faults without reference to sensor faults, making these methods less applicable.

This paper aims to design an adaptive observer for nonlinear systems described by Takagi-Sugeno with non-measurable decision variables and affected by the simultaneous actuator and sensor faults under unknown disturbances. The proposed technic is based on a proportional-integral observer with unknown input. Firstly, the multi-model structure is used to represent the nonlinear system by interpolating local linear models. Then, a mathematical transformation is used to rewrite the sensor fault as an actuator fault. The exponential stability conditions are synthesized using the Lyapunov function and L2 optimization and formulated in LMIs. To improve the efficacy of the proposed method, it is applied to a CSTR.

The main contributions of this work are: 1) using the multi-model approach to circumvent the nonlinearity of the system; 2) extending the adaptive observer to state and faults estimation for TS systems with unmeasurable premise variables; 3) the considered system is affected by the simultaneous actuator and sensor faults under unknown disturbances; 4) new stability conditions are obtained based on the Lyapunov theory and L2 optimization, and formulated in terms of LMIs which can be solved easily in Matlab LMI Toolbox; finally 5) application of the proposed method on a CSTR.

This paper is organized as follows: the Takagi-Sugeno approach is presented in Section 2 , Section 3 concerns the problem statement and the adaptive observer synthesis, Section 4 focuses on the application to the CSTR system, finally, the conclusion is given in section 5 .

\section{Takagi-Sugeno multi-model approach}

The Takagi-Sugeno multi-model method aims to divide the nonlinear system into an interpolation of linear sub-models. The multiple models are presented in the following form:

$\left\{\begin{array}{l}\dot{x}(t)=\sum_{i=1}^{l} \mu_{i}(\xi(t))\left(A_{i} x(t)+B_{i} u(t)\right) \\ y(t)=\sum_{i=1}^{l} \mu_{i}(\xi(t)) C_{i} x(t)\end{array}\right.$ 
where $x(t) \in \mathbb{R}^{n}$ is the state vector, $y(t) \in \mathbb{R}^{p}$ is the output, $u(t) \in \mathbb{R}^{m}$ is the control. $l$ is the number of local models, $A_{i} \in \mathbb{R}^{n \times n}, B_{i} \in \mathbb{R}^{n \times m}$ and $C_{i} \in \mathbb{R}^{p \times n}$ are constant matrices of appropriate dimensions. $\mu_{i}(\xi(t))$ are the activation functions which define the activation degree of a local model and $\xi(t)$ is the decision variable. These functions satisfy the following convexity property:

$\left\{\begin{array}{c}\sum_{i=1}^{l} \mu_{i}(\xi(t))=1, \forall t \\ 0 \leq \mu_{i}(\xi(t)) \leq 1, \forall i=1 \ldots l\end{array}\right.$

\section{The design of the adaptive observer}

This section discusses the synthesis of an adaptive observer for a Takagi-Sugeno multimodel system with non-measurable variables decision with simultaneous actuator and sensor faults.

\subsection{Problem statement.}

Let us consider a TS multi-model affected by actuator and sensor faults as follows:

$\left\{\begin{array}{l}\dot{x}(t)=\sum_{i=1}^{l} \mu_{i}(x(t))\left(A_{i} x(t)+B_{i} u(t)+E_{i} f_{a}(t)+\mathrm{wd}(\mathrm{t})\right) \\ y(t)=C x(t)+S f_{s}(t)+\omega \mathrm{d}(\mathrm{t})\end{array}\right.$

where $f_{a}(t)$ and $f_{s}(t)$ are respectively the actuator and sensor faults, and $\mathrm{d}(\mathrm{t})$ is an unknown disturbance. $E_{i}$ and $S$ are the faults distribution matrices that are supposed to be known.

A new state $q(t)$ is introduced in order to rewrite the sensor faults as actuator faults.

$\dot{q}(t)=\sum_{i=1}^{l} \mu_{i}(x(t))(-D q(t)+D y(t))$

The augmented system is presented as:

$\left\{\begin{array}{l}\dot{X}(t)=\sum_{i=1}^{l} \mu_{i}(x(\mathrm{t}))\left(\bar{A}_{i} x(t)+\bar{B}_{i} u(t)+\bar{E}_{i} f(t)+\bar{w} d(t)\right) \\ Y(t)=\bar{C} x(t)\end{array}\right.$

where $\bar{w}=\left[\begin{array}{cc}\mathrm{w} & 0 \\ 0 & D \omega\end{array}\right]$

The states of the considered system are supposed not completely measurable. Therefore. (5) is rewritten as a multi-model with estimated variables decision.

$\left\{\begin{array}{l}\dot{X}(t)=\sum_{i=1}^{l} \mu_{i}(\hat{\mathrm{x}}(\mathrm{t}))\left(\bar{A}_{i} x(t)+\bar{B}_{i} u(t)+\bar{E}_{i} f(t)+\bar{w} d(t)\right)+\Delta(t) \\ Y(t)=\bar{C} x(t)\end{array}\right.$

where $\hat{\mathrm{x}}(\mathrm{t})$ is the estimated $x(\mathrm{t})$ by the proposed observer and $\Phi(t)$ given by:

$\Delta(t)=\sum_{i=1}^{l}\left(\mu_{\mathrm{i}}(x(\mathrm{t}))-\mu_{\mathrm{i}}(\hat{\mathrm{x}}(\mathrm{t}))\right)\left(\bar{A}_{i} x(t)+\bar{B}_{i} u(t)+\bar{E}_{i} f(t)+\bar{w} d(t)\right)$

The system (6) can be rewritten as a faulty multi-model with unknown input.

$\left\{\begin{array}{l}\dot{X}(t)=\sum_{i=1}^{l} \mu_{i}(\hat{\mathrm{x}}(\mathrm{t}))\left(\bar{A}_{i} x(t)+\bar{B}_{i} u(t)+\bar{E}_{i} f(t)\right)+\bar{W} \phi(t) \\ Y(t)=\bar{C} x(t)\end{array}\right.$

with

$\overline{A_{i}}=\left[\begin{array}{cc}A_{i} & 0 \\ D C & -D\end{array}\right] ; \bar{B}_{i}=\left[\begin{array}{c}B_{i} \\ 0\end{array}\right] ; \bar{E}_{i}=\left[\begin{array}{cc}E_{i} & 0 \\ 0 & D S\end{array}\right] ; \bar{C}=\left[\begin{array}{cc}C & 0 \\ 0 & I\end{array}\right] ; X(t)=\left[\begin{array}{l}x(t) \\ q(t)\end{array}\right] ; f(t)=\left[\begin{array}{l}f_{a}(t) \\ f_{s}(t)\end{array}\right] ;$

$\bar{W}=\left[\begin{array}{ll}\bar{W} & \mathrm{I}\end{array}\right] ; \phi(t)=\left[\begin{array}{l}d(t) \\ \Delta(t)\end{array}\right]$.

3.2. Design and stability conditions of the adaptive observer.

The proposed observer is structured as follows: 
$\left\{\begin{array}{l}\dot{z}(t)=\sum_{i=1}^{l} \mu_{i}(\hat{x}(t))\left(R_{i} z(t)+T \bar{B}_{i} u(t)+T \bar{E}_{i} \hat{f}(t)+K_{P i} y(t)\right) \\ \dot{\hat{f}}(t)=\sum_{i=1}^{l} \mu_{i}(\hat{x}(t)) K_{I i}(Y-\bar{C} \hat{X}) \\ \hat{X}(t)=z(t)+H Y(t)\end{array}\right.$

where $\hat{x}(t)$ is the estimated system state, $z(t)$ is an intermediate variable and $\hat{\mathrm{f}}(t)$ is the estimated faults. The variables $R_{i}, T, K_{P i}, K_{I i}$ and $H$ are the observer gains.

Stability conditions are obtained in the following theorem.

\section{Theorem}

The convergence of the state estimation error between the system (6) and the adaptive observer (9) is verified and the L2-gain of the transfer from $\Phi(t)$ to the estimation error is bounded if there exists $P=P^{T}>0$ and matrix $M_{i}$ and positive scalars $\bar{\gamma}$ the solution to the following optimization problem:

$$
\begin{aligned}
& \min \bar{\gamma} \\
& \quad \mathrm{P}, M_{i}, \bar{\gamma}
\end{aligned}
$$

and the following conditions hold:

$$
\left[\begin{array}{cc}
\bar{A}_{a i}^{T} P+P \bar{A}_{a i}-\bar{C}_{a}{ }^{T} M_{i}^{T}-M_{i} \bar{C}_{a}+2 \alpha P+I & P \Gamma \\
\Gamma^{T} P & -\bar{\gamma} I
\end{array}\right]<0 i=1, \ldots, l
$$

with $\alpha>0$ and:

$$
\begin{gathered}
{\left[\begin{array}{cc}
T & H
\end{array}\right]=\left[\begin{array}{c}
I_{n} \\
\bar{C}
\end{array}\right]^{+}} \\
{\left[\begin{array}{c}
F_{i} \\
K_{I i}
\end{array}\right]=\bar{K}_{i}=X^{-1} M_{i}} \\
R_{i}=\mathrm{T} \bar{A}_{i}-F_{i} \bar{C} \\
K_{P i}=F_{i}+R_{i} M
\end{gathered}
$$

and the L2-gain of the transfer is calculated by: $\gamma=\sqrt{\bar{\gamma}}$

\section{Proof}

$e(t)$ and $e_{f}(t)$ represent the state and the fault estimation error respectively:

$$
e(t)=X(t)-\hat{X}(t)
$$

$$
e_{f}(t)=f(t)-\hat{f}(t)
$$

The dynamics of the state estimation error is obtained as:

$$
\begin{aligned}
& \dot{e}(t)=\dot{X}(t)-\dot{\hat{X}}(t)=\sum_{i=1}^{l} \mu_{i}(\hat{x}(t))\left(\left(P \bar{A}_{i}-R_{i}-F_{i} \bar{C}\right) X(t)+\left(\Omega \bar{B}_{i}-T \bar{B}_{i}\right) u(t)+\right. \\
& \left.\left.\left(\Omega \bar{E}_{i}-T \bar{E}_{i}\right) f(t)+\Omega \bar{W} \Phi(t)+R_{i} e(t)\right)+T \bar{E}_{i} e_{f}(t)\right)
\end{aligned}
$$

where

$\Omega=I-H \bar{C}$

$F_{i}=K_{P i}-R_{i} H$

If the following conditions are verified

$R_{i}=\Omega \bar{A}_{i}-F_{i} \bar{C}$

$\Omega=T$

The state estimation error can be rewritten as:

$\dot{e}(t)=R_{i} e(t)+T \bar{E}_{i} e_{f}(t)+\Omega \bar{W} \Phi(t)$

The dynamics of the fault estimation error is obtained by:

$\dot{e}_{f}=-\dot{\hat{\bar{u}}}(t)=-\sum_{i=1}^{l} \mu_{i}(\hat{x}(t)) K_{I i}(Y-\bar{C} \hat{X})=-\sum_{i=1}^{l} \mu_{i}(\hat{x}(t)) K_{I i} \bar{C} e(t)$

The augmented form of $\dot{e}(t)$ and $\dot{e}_{f}(t)$ is given by: 
$\dot{e}_{a}(t)=\left[\begin{array}{cc}R_{i} & T \bar{E}_{i} \\ -K_{I i} \bar{C} & 0\end{array}\right] e_{a}(t)+\left[\begin{array}{c}\Omega \bar{W} \\ 0\end{array}\right] \Phi(\mathrm{t})$

where $e_{a}(t)=\left[\begin{array}{c}e(t) \\ e_{u}(t)\end{array}\right]$

The estimation error $e_{a}(t)$ is reduced to:

$\dot{e}_{a}(t)=\left(\bar{A}_{a i}-\bar{K}_{i} \bar{C}_{a}\right) e_{a}(t)+\Gamma \Phi(t)$

with: $\bar{A}_{a i}=\left[\begin{array}{cc}\Omega \bar{A}_{i} & T \bar{E}_{i} \\ 0 & 0\end{array}\right] ; \bar{K}_{i}=\left[\begin{array}{c}F_{i} \\ K_{I i}\end{array}\right] ; \bar{C}_{a}=\left[\begin{array}{ll}\bar{C} & 0\end{array}\right] ; \Gamma=\left[\begin{array}{c}\Omega \bar{W} \\ 0\end{array}\right]$

Tacking account of (18) and (21), we can write the following equation:

$\mathrm{I}=\mathrm{T}+M \bar{C}=\left[\begin{array}{ll}T & M\end{array}\right]\left[\begin{array}{c}I_{n} \\ \bar{C}\end{array}\right]$

The matrix $I_{n}$ is an identity matrix with appropriate dimension. Therefore, the gains $T$ an $H$ can be obtained as:

$\left[\begin{array}{ll}T & H\end{array}\right]=\left[\begin{array}{c}I_{n} \\ \bar{C}\end{array}\right]^{+}$

where $\left[\begin{array}{c}I_{n} \\ \bar{C}\end{array}\right]^{+}$the pseudo-inverse of $\left[\begin{array}{c}I_{n} \\ \bar{C}\end{array}\right]$.

Let's consider the following Lyapunov function:

$V(t)=e_{a}^{T}(t) P e_{a}(t)$

$e_{a}(t)$ converge exponentially to zero if:

$\exists X=X^{T}>0, \alpha>0: \dot{V}(t)+2 \alpha V(t)<0$

The time derivative of $V(t)$ is calculated as:

$\dot{V}(t)=\sum_{\mathrm{i}=1}^{\mathrm{l}} \mu_{\mathrm{i}}(\hat{\mathrm{x}}(\mathrm{t}))\left(e_{a}^{T}(t)\left(\left(\bar{A}_{a i}-\bar{K}_{i} \bar{C}_{a}\right)^{T} P+X\left(\bar{A}_{a i}-\bar{K}_{i} \bar{C}_{a}\right)+2 \alpha P\right) e_{a}(t)+\right.$

$\left.\left.\Phi^{T}(t) \Gamma^{T} P e_{a}(t)+e_{a}^{T}(t) \mathrm{P} \Gamma \Phi(t)\right)\right)$

The minimization of L2-gain of transfer from $\Phi(t)$ to the estimation error $e_{a}(t)$ is given by:

$$
\frac{\left\|e_{a}(t)\right\|_{2}}{\|\Phi(t)\|_{2}}<\gamma,\|\Phi(t)\|_{2} \neq 0
$$

The estimation error converges exponentially toward zero and the L2-gain of transfer from $\Phi(t)$ to the estimation error $e_{a}(t)$ is bounded if the following condition is verified:

$\dot{V}(t)+e_{a}^{T}(t) e_{a}(t)-\gamma^{2} \Phi^{T}(t) \Phi(t)+2 \alpha V(t)<0$

By replacing $V(t)$ in (31) by its definition, the following inequality is obtained:

$\left[\begin{array}{cc}\bar{A}_{a i}^{T} P+P \bar{A}_{a i}-\bar{C}_{a}{ }^{T} M_{i}^{T}-M_{i} \bar{C}_{a}+2 \alpha P+I & P \Gamma \\ \Gamma^{T} P & -\bar{\gamma} I\end{array}\right]<0 i=1, \ldots, l$

\section{Simulation results}

\subsection{CSTR system description.}

Consider an isothermal CSTR with series-parallel reaction (Van der Vusse reaction) described by the following reactions [35]:

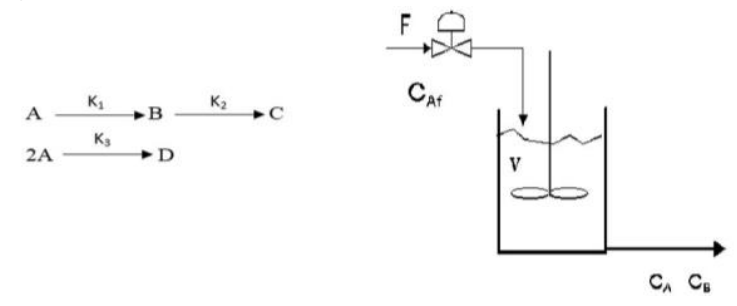

Figure 1. Isothermal Continuous Stirred-Tank Reactor. 
The volume of the reactor $V$ is assumed constant, and the feed stream contains only the component $A . k_{1}, k_{2}$ and $k_{3}$ are the reaction rate constants, $C_{A f}$ is the inlet concentration of the product $A$ which will be converted to the desired product B.

The following set of differential equations describe the van der Vusse reaction scheme in an isothermal CSTR with actuator and sensor faults under unknown disturbances:

$\left\{\begin{array}{l}\frac{d C_{A}}{d t}=\left(\frac{F}{V}+f_{a}(t)\right)\left(C_{A f}-C_{A}\right)-k_{1} C_{A}-k_{3} C_{A}^{2}+\mathrm{w}_{1} \mathrm{~d}(\mathrm{t}) \\ \frac{d C_{B}}{d t}=-\left(\frac{F}{V}+f_{a}(t)\right) C_{B}-k_{1} C_{A}-k_{2} C_{B}+\mathrm{w}_{2} \mathrm{~d}(\mathrm{t})\end{array}\right.$
$y(t)=C_{B}+S f_{S}(t)+\omega \mathrm{d}(\mathrm{t})$

The equations for $C_{C}$ and $C_{D}$ are neglected because $C_{B}$ is independent of them. The manipulated input in this system is the dilution rate $\frac{F}{V}$, and the controlled output variable is the product concentration $C_{B}$. The state representation of the system is as follows:

$\left[\begin{array}{c}\dot{C}_{A} \\ \dot{C}_{B}\end{array}\right]=\left[\begin{array}{cc}-k_{1}-k_{3} C_{A} & 0 \\ k_{1} & -k_{2}\end{array}\right]\left[\begin{array}{c}C_{A} \\ C_{B}\end{array}\right]+\left[\begin{array}{c}C_{A f}-C_{A} \\ -C_{B}\end{array}\right] \frac{F}{V}+\left[\begin{array}{c}C_{A f}-C_{A} \\ -C_{B}\end{array}\right] f_{a}(t)+\left[\begin{array}{l}w_{1} \\ w_{2}\end{array}\right] \mathrm{d}(\mathrm{t})$

Equation (36) can be written as:

$\left\{\begin{array}{l}\dot{x}(t)=A(x(t)) x(t)+B(x(t)) u(t)+B(x(t)) f_{a}(t)+w d(t) \\ y(t)=C x(t)+S f_{s}(t)+\omega \mathrm{d}(\mathrm{t})\end{array}\right.$

where $w(t)=\left[\begin{array}{l}W_{1} \\ w_{2}\end{array}\right]$

The parameters values are given in Table 1.

Table 1. Parameters values.

\begin{tabular}{c|c} 
Parameters & Values \\
\hline$k_{1}$ & $\frac{5}{6} \mathrm{~min}^{-1}$ \\
\hline$k_{2}$ & $\frac{5}{3} \mathrm{~min}^{-1}$ \\
\hline$k_{3}$ & $\frac{1}{6} \mathrm{~mol} / \mathrm{l} . \mathrm{min}$ \\
\hline$C_{A f}$ & $10 \mathrm{~mol} / \mathrm{l}$
\end{tabular}

\subsection{Isothermal CSTR Takagi-Sugeno model design.}

In the state representation (37), $C_{A}$ and $C_{B}$ are nonlinear terms, so we make them as our fuzzy variables. Generally, they are denoted by $z_{i}(t)$, and are known as premise variables.

Therefore, $z_{1}(t)=C_{A}$ and $z_{2}(t)=C_{B}, z_{i}(t)$ can be represented by membership functions $F_{i}, f_{i}$, the maximums $M_{i}$ and the minimums $m_{i}$ of $z_{i}(t)$ as follows:

$\left\{\begin{array}{l}z_{1}(t)=F_{1}\left(z_{1}(t)\right) M_{1}+f_{1}\left(z_{1}(t)\right) m_{1} \\ z_{2}(t)=F_{2}\left(z_{2}(t)\right) M_{2}+f_{2}\left(z_{2}(t)\right) m_{2}\end{array}\right.$

According to (38), the membership functions can be calculated as follows:

$\begin{cases}F_{1}\left(z_{1}(t)\right)=\frac{z_{1}(t)-m_{1}}{M_{1}-m_{1}}, & f_{1}\left(z_{1}(t)\right)=\frac{M_{1}-z_{1}(t)}{M_{1}-m_{1}} \\ F_{2}\left(z_{2}(t)\right)=\frac{z_{2}(t)-m_{2}}{M_{2}-m_{2}}, & f_{2}\left(z_{2}(t)\right)=\frac{M_{2}-z_{2}(t)}{M_{2}-m_{2}}\end{cases}$

and the activations function are: 
$\left\{\begin{array}{l}\mu_{1}(z(t))=F_{1}\left(z_{1}(t)\right) F_{2}\left(z_{2}(t)\right) \\ \mu_{2}(z(t))=f_{1}\left(z_{1}(t)\right) F_{2}\left(z_{2}(t)\right) \\ \mu_{3}(z(t))=F_{1}\left(z_{1}(t)\right) f_{2}\left(z_{2}(t)\right) \\ \mu_{4}(z(t))=f_{1}\left(z_{1}(t)\right) f_{2}\left(z_{2}(t)\right)\end{array}\right.$

The subsystems are determined as:

$$
\left\{\begin{array}{l}
\dot{x}(t)=\sum_{i=1}^{4} \mu_{i}(z(t))\left[A_{i} x(t)+B_{i} u(t)+E_{i} f_{a}(t)+w d(t)\right] \\
y(t)=\mathrm{Cx}(\mathrm{t})+S f_{S}(t)+\omega \mathrm{d}(\mathrm{t})
\end{array}\right.
$$

where $E_{i}=B_{i}$ and matrices $A_{i}, B_{i}$ and $\mathrm{C}$ are given in Table 2 .

Table 2. Sub-models of CSTR.

\begin{tabular}{|c|c|c|c|}
\hline Sub-model $i$ & $A_{i}$ & $B_{i}$ & C \\
\hline 1 & {$\left[\begin{array}{c}-k_{1}-k_{3} M_{1} \\
k_{1}\end{array}\right.$} & {$\left[\begin{array}{c}C_{A f}-M_{1} \\
-M_{2}\end{array}\right]$} & \multirow{4}{*}[\begin{array}{ll}{0}&{1}\end{array}]{} \\
\hline 2 & {$\left[\begin{array}{c}-k_{1}-k_{3} m_{1} \\
k_{1}\end{array}\right.$} & {$\left[\begin{array}{c}C_{A f}-m_{1} \\
-M_{2}\end{array}\right]$} & \\
\hline 3 & {$\left[\begin{array}{c}-k_{1}-k_{3} M_{1} \\
k_{1}\end{array}\right.$} & {$\left[\begin{array}{c}C_{A f}-M_{1} \\
-m_{2}\end{array}\right]$} & \\
\hline 4 & {$\left[\begin{array}{c}-k_{1}-k_{3} m_{1} \\
k_{1}\end{array}\right.$} & {$\left[\begin{array}{c}C_{A f}-m_{1} \\
-m_{2}\end{array}\right]$} & \\
\hline
\end{tabular}

\subsection{Simulations results and discussions.}

The acting faults appear and disappear during an interval time as follows:

$f_{a}=\left\{\begin{array}{cc}0.4, & 20 \leq t \leq 50 \\ 0 & \text { elsewhere }\end{array} ; f_{s}=\left\{\begin{array}{cr}0.3 \sin (0.06 \pi t), & 35 \leq t \leq 70 \\ 0 & \text { elsewhere }\end{array}\right.\right.$

The unknown disturbances are presented by zero-mean noise with standard deviations equal to 0.05 . The distribution matrices $S, W$ and $\omega$ are given by:

$$
S=0.75 ; W=1.2 \text { and } \omega=\left[\begin{array}{l}
0.8 \\
0.9
\end{array}\right]
$$

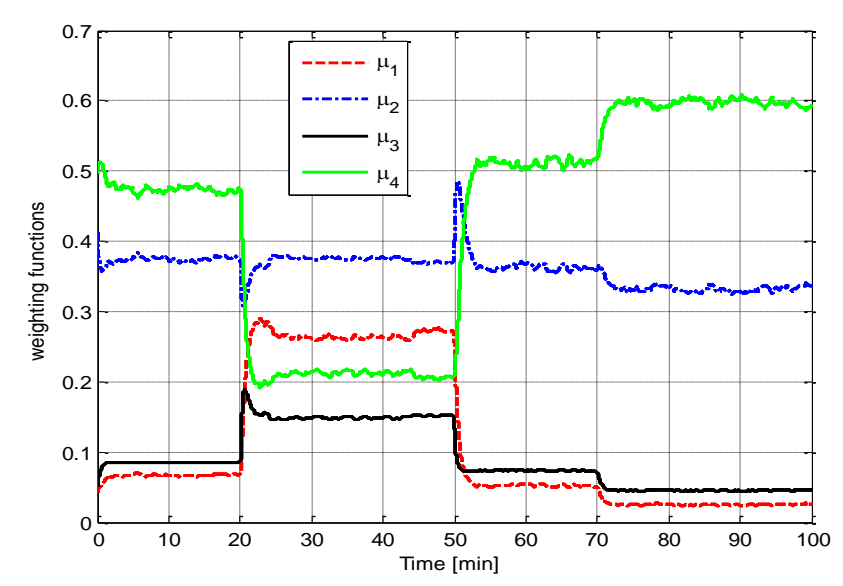

Figure 2. Time-evolution of the weighting functions.

The LMI elaborated in the proposed theorem is solved using the Matlab Yalmip toolbox, and the gains of the adaptive observer are calculated. States and faults estimations are presented in Figure 5 and Figure 6. 


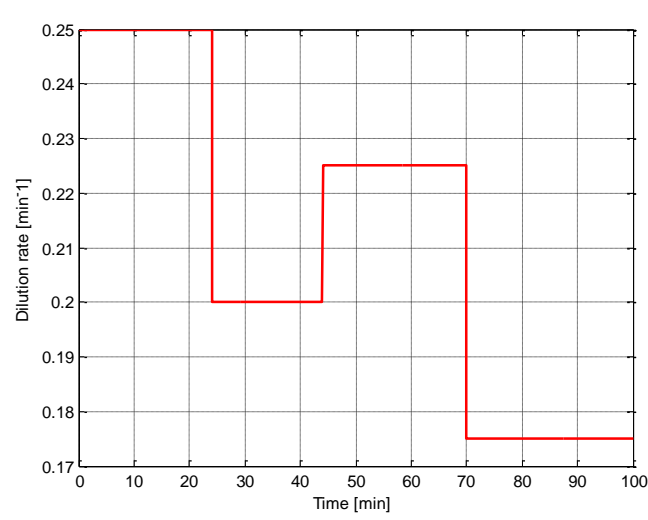

Figure 3. The control input.

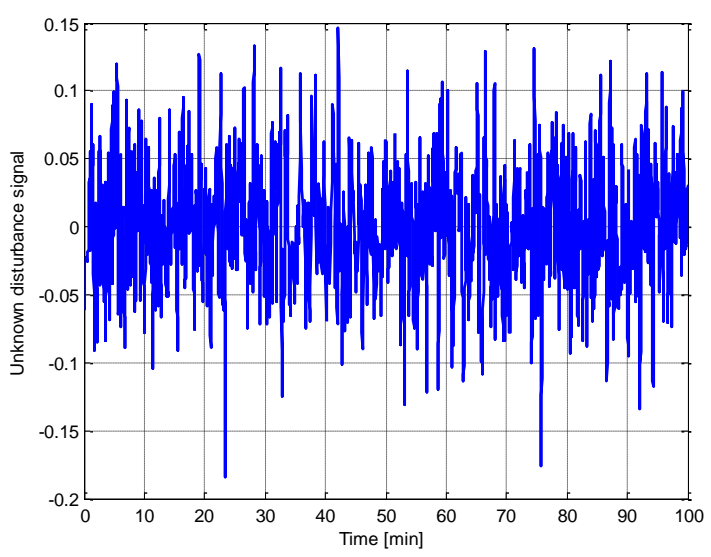

Figure 4. Unknown disturbance signal.
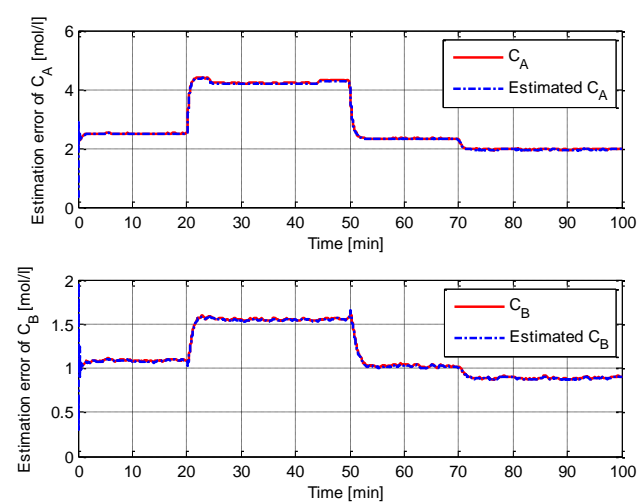

Figure 5. States estimation.
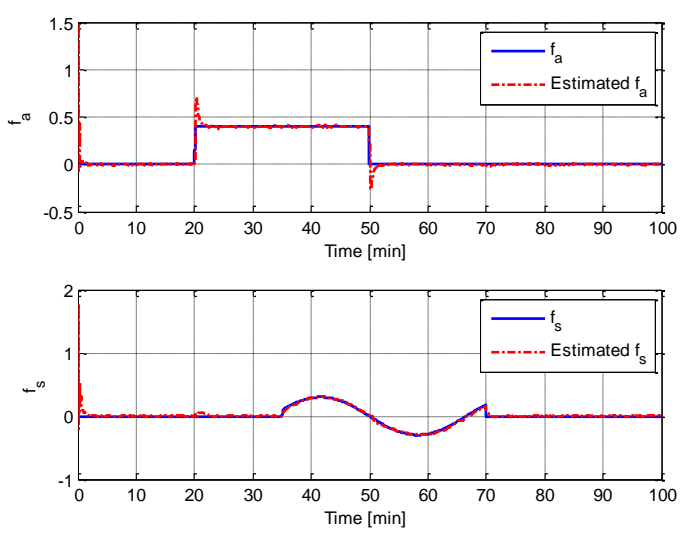

Figure 6. Actuator and sensor faults with their estimates.

The weighting functions for each sub-model are presented in Figure 2, always close to the dynamic behavior of the nonlinear system according to the considered operating regimes. Figure 3 and Figure 4 present the control input and the unknown disturbance signals, respectively. Figure 5 shows that from moment zero, the estimated states converge rapidly to their real signals. Figure 6 presents a good estimation of the actuator and sensor faults, respectively. According to the simulation results, we can clearly see that the proposed observer ensures the estimation of states and the simultaneous actuator and sensor faults despite unknown disturbances.

\section{Conclusions}

In this work, an adaptive observer is proposed to estimate the states and simultaneous actuator and sensor faults of a CSTR with unknown disturbances. Firstly, the Takagi-Sugeno method is proposed to represent the complex nonlinear system as a multi-model with unmeasurable variables decision. Secondly, a mathematical transformation is used to rewrite all faults as actuator faults. The exponential stability conditions are studied with Lyapunov theory and L2 optimization and given in linear matrix inequalities form. Finally, a simulation example is carried out on a CSTR. From the results, states, actuator, and sensor faults are successfully estimated in the presence of the unknown disturbances by minimizing the effects of these last. 


\section{Funding}

This research received no external funding.

\section{Conflicts of Interest}

The authors declare no conflict of interest.

\section{References}

1. Luenberger, D. G. Observers for multivariable systems. IEEE Trans. Automat. Contr 1966, 11, 190-197, https://doi.org/10.1109/TAC.1966.1098323.

2. Thau, E. E. Observing the state of nonlinear systems. International Journal of Contro 1973, 17, 471-479, https://doi.org/10.1080/00207177308932395.

3. Mansouri, M.; Nounou, H.; Nounou, M. State estimation of a chemical reactor process model-a comparative study. IEEE - 10th International Multi-Conferences on Systems, Signals \& Devices 2013, 1-6, https://doi.org/10.1109/ssd.2013.6563998.

4. Geetha, M.; Jerome, J.; Kumar, P. A.; Anadhan, K. Comparative performance analysis of extended Kalman filter and neural observer for state estimation of continuous stirred tank reactor. IEEE - 2013 Fourth International Conference on Computing, Communications and Networking Technologies (ICCCNT) 2013, 17, https://doi.org/10.1109/ICCCNT.2013.6726715.

5. Thirumarimurugan, M.; Anusharani, V.; Prabhakaran, D. state estimation in a continuous stirred tank reactor (CSTR) using observers. Journal of Electrical Engineering 2021, 21, 10-10, http://www.jee.ro/index.php/jee/article/view/WB1558697277W5ce7d53de7b08.

6. Takagi, T.; Sugeno, M. Fuzzy identification of systems and its applications to model and control. IEEE Transactions on Systems, Man, and Cybernetics 1985, 15, 116-132, https://doi.org/10.1109/TSMC.1985.6313399.

7. Martínez-García, C.; Puig, V.; Astorga-Zaragoza, C. M.; Madrigal-Espinosa, G.; Reyes-Reyes, J. Estimation of actuator and system faults via an unknown input interval observer for takagi-sugeno systems. Processes 2020, 8, 61, https://doi.org/10.3390/pr8010061.

8. Feng, X.; Wang, Y. Fault estimation based on sliding mode observer for Takagi-Sugeno fuzzy systems with digital communication constraints. Journal of the Franklin Institute 2020, 357, 569-588, https://doi.org/10.1016/j.jfranklin.2019.09.020.

9. Zhao, D.; Lam, H. K.; Li, Y.; Ding, S. X.; Liu, S. A novel approach to state and unknown input estimation for Takagi-Sugeno fuzzy models with applications to fault detection. IEEE Transactions on Circuits and Systems I: Regular Papers 2020, 67, 2053-2063, https://doi.org/10.1109/TCSI.2020.2968732.

10. Garbouj, Y.; Dinh, T. N.; Raissi, T.; Zouari, T.; Ksouri, M. Optimal interval observer for switched TakagiSugeno systems: an application to interval fault estimation. IEEE Transactions on Fuzzy Systems 2020, 29, 2296-2309, https://doi.org/10.1109/TFUZZ.2020.2997333.

11. Djeddi, A.; Dib, D.; Azar, A. T.; Abdelmalek, S. Fractional order unknown inputs fuzzy observer for TakagiSugeno systems with unmeasurable premise variables. Mathematics 2019, 7, 984, https://doi.org/10.3390/math7100984.

12. Sun, S.; Zhang, H.; Han, J.; Liang, Y. A novel double-level observer-based fault estimation for TakagiSugeno fuzzy systems with unknown nonlinear dynamics. Transactions of the Institute of Measurement and Control 2019, 41, 3372-3384, https://doi.org/10.1177/0142331219826655.

13. You, F.; Cheng, S.; Zhang, X.; Chen, N. Robust fault estimation for Takagi-Sugeno fuzzy systems with state time-varying delay. International Journal of Adaptive Control and Signal Processing 2019, 34, 141-150, https://doi.org/10.1002/acs.3073.

14. Chaves, E. R.; de A. Dantas, A.F.; Maitelli, A. L. Unknown Input Observer-based Actuator and Sensor Fault Estimation Technique for Uncertain Discrete Time Takagi-Sugeno Systems. International Journal of Control Automation and Systems 2021, 19, 2444-2454, https://doi.org/10.1007/s12555-020-0170-x.

15. Jia, Q. ; Wu, L. ; Li, H. Robust Actuator Fault Reconstruction for Takagi-Sugeno Fuzzy Systems with Timevarying Delays via a Synthesized Learning and Luenberger Observer. International Journal of Control, Automation and Systems 2021, 19, 799-809, http://dx.doi.org/10.1007/s12555-019-0747-4.

16. Zhang, W.; Wang, Z.; Raïssi, T.; Wang, Y.; Shen, Y. A state augmentation approach to interval fault estimation for descriptor systems. European Journal of Control 2020, 51, 19-29, http://dx.doi.org/10.1016/j.ejcon.2019.06.006.

17. Yunfei, M.; Huaguang, Z.; Hanguang, S.; He, R. Unknown input observer synthesis for discrete-time T-S fuzzy singular systems with application to actuator fault estimation. Nonlinear Dynamics 2020, 100, 33993412, https://doi.org/10.1007/s11071-020-05630-6.

18. Wang, X.; Fei, Z.; Wang, T.; Yang, L. Dynamic event-triggered actuator fault estimation and accommodation for dynamical systems. Information Sciences 2020, 525, 119-133, https://doi.org/10.1016/j.ins.2020.03.016. 
19. Li, Z.; Zhang, H.; Yan, H.; Lam, H. K.; Huang, C. Aperiodic Sampled-Data Takagi-Sugeno Fuzzy Extended State Observer for A Class of Uncertain Nonlinear Systems with External Disturbance and Unmodeled Dynamics. IEEE Transactions on Fuzzy Systems 2021, 1-1, https://doi.org/10.1109/TFUZZ.2021.3090923.

20. Zare, K.; Shasadeghi, M.; Izadian, A.; Niknam, T.; Asemani, M. H. Switching TS fuzzy model-based dynamic sliding mode observer design for non-differentiable nonlinear systems. Engineering Applications of Artificial Intelligence 2020, 96,103990, https://doi.org/10.1016/j.engappai.2020.103990.

21. Chen, Y.; Zhu, X.; Gu, J. Fault Estimation and Compensation for Fuzzy Systems with Sensor Faults in LowFrequency Domain. In International Conference in Communications, Signal Processing, and Systems Springer, Singapore 2020, 799-807, https://doi.org/10.1007/978-981-15-8411-4_106

22. Zhang, K.; Jiang, B.; Ding, S. X.; Zhou, D. Robust asymptotic fault estimation of discrete-time interconnected systems with sensor faults. IEEE transactions on cybernetics 2020, 1-10, https://doi.org/10.1109/TCYB.2020.2986386.

23. Bouassem, K.; El Assoudi, A.; Soulami, J.; El Yaagoubi, H. Unknown Input Observer Design for a Class of Linear Descriptor Systems. In E3S Web of Conferences- EDP Sciences 2021, 229, 01019, https://doi.org/10.1051/e3sconf/202122901019.

24. Ouarid, K.; El Assoudi, A.; El Yaagoubi, H.; Essabre, M. Fuzzy Observer Design for Fault Estimation for Discrete-time Takagi-Sugeno Singular Models with Unmeasurable Premise Variables. International Journal of Intelligent Engineering and Systems 2021, 14, 514-523, https://doi.org/10.22266/ijies2021.0831.45.

25. Heras-Cervantes, M.; Téllez-Anguiano, A. D. C.; Anzurez-Marín, J.; Espinosa-Juárez, E. Analysis and Comparison of Fuzzy Models and Observers for DC-DC Converters Applied to a Distillation Column Heating Actuator. Mathematical and Computational Applications 2020, 25, 55, https://doi.org/10.3390/mca25030055.

26. Márquez-Vera, M. A.; Rodríguez-Romero, A.; Márquez-Vera, C. A.; Ramos-Téllez, K. R. Interval Type-2 Fuzzy Observers Applied in Biodegradation. International Journal of Robotics and Control Systems 2021, 1, 145-158, https://doi.org/10.31763/ijrcs.v1i2.344.

27. Hosseini, I.; Fiacchini, M.; Karimaghaee, P.; Khayatian, A. Optimal reset unknown input observer design for fault and state estimation in a class of nonlinear uncertain systems. Journal of the Franklin Institute 2020, 357, 2978-2996, https://doi.org/10.1016/j.jfranklin.2019.12.008.

28. Li, S.; Aitouche, A.; Wang, H.; Christov, N. Sensor fault estimation of PEM fuel cells using Takagi Sugeno fuzzy model. International Journal of Hydrogen Energy 2020, 45, 11267-11275, https://doi.org/10.1016/j.ijhydene.2019.01.100.

29. Khedher, A.; Elleuch, I.; BenOthman, K. Adaptive Proportional Integral Observer Design for Interval Type 2 Takagi-Sugeno Fuzzy Systems. Mathematical Problems in Engineering 2021, 2021, Article ID 5317391, https://doi.org/10.1155/2021/5317391

30. Elleuch, I.; Khedher, A.; Othman, K. B. State and faults estimation based on proportional integral sliding mode observer for uncertain Takagi-Sugeno fuzzy systems and its application to a turbo-reactor. International Journal of Fuzzy Systems 2017, 19, 1768-1781, https://doi.org/10.1007/s40815-017-0365-3.

31. Guzman, J.; López-Estrada, F. R.; Estrada-Manzo, V.; Valencia-Palomo, G. Actuator fault estimation based on a proportional-integral observer with nonquadratic Lyapunov functions. International Journal of Systems Science 2021, 52, 1-14, https://doi.org/10.1080/00207721.2021.1873451.

32. Zhang, X.; Feng, X.; Mu, Z.; Wang, Y. State and fault estimation for nonlinear recurrent neural network systems: Experimental testing on a three-tank system. The Canadian Journal of Chemical Engineering 2020, 98, 1328-1338, https://doi.org/10.1002/cjce.23714.

33. Wang, Z. P.; Wu, H. N.; Chadli, M. $\mathrm{H}_{\infty}$ Sampled-Data Fuzzy Observer Design for Nonlinear Parabolic PDE Systems. IEEE Transactions on Fuzzy Systems 2021, 29, 1262-1272, https://doi.org/10.1109/TFUZZ.2020.2973943.

34. Asadi, S.; Khayatian, A.; Dehghani, M.; Vafamand, N.; Khooban, M. H. Robust sliding mode observer design for simultaneous fault reconstruction in perturbed Takagi-Sugeno fuzzy systems using non-quadratic stability analysis. Journal of Vibration and Control 2020, 26, 1092-1105, https://doi.org/10.1177/1077546319891693.

35. Nagarajan, M.; Asokan, A.; Manikandan, M.; Sivakumar, D. Concentration Control of Isothermal CSTR using Particle Swarm Optimization based FOPID Controller. Middle-East Journal of Scientific Research 2016, 24, 967-971, https://www.idosi.org/mejsr/mejsr24(3)16/68.pdf. 